\title{
The impact of disease activity on BMI in paediatric inflammatory bowel disease
}

\author{
T. M. Hunt ${ }^{1}$, A. E. Wiskin ${ }^{1}$, N. A. Afzal ${ }^{1}$, S. A. Wootton ${ }^{2}$ and R. M. Beattie ${ }^{1}$ \\ ${ }^{1}$ Paediatric Gastroenterology Unit, Southampton General Hospital, SO16 6YD and ${ }^{2}$ Institute of Human Nutrition, University \\ of Southampton SO16 6YD
}

Twenty-five percent of Inflammatory Bowel Disease (IBD) presents in childhood. It is well recognised that nutritional impairment is common at diagnosis in Crohn's disease (CD) but appears less prevalent in Ulcerative Colitis (UC) ${ }^{(1)}$. There is a lack of clinical tools used in clinical practice to detect undernutrition. BMI is a simple tool which adjusts weight for variations in height, although does not accurately reflect body composition. Previous studies have examined changes in body composition in active IBD although no studies have directly related BMI to disease activity. The study hypothesis was that BMI would decrease with increasing disease activity in both $\mathrm{CD}$ and UC.

Measures of height, weight and disease activity were collected prospectively from children attending the paediatric gastroenterology unit at Southampton General Hospital. Disease activity was scored using the Paediatric Disease Activity Index (PCDAI) for CD ${ }^{(2)}$ and the Simple Colitis Activity Index (SCAI) for $\mathrm{UC}^{(3)}$. All children were categorised according to the Montreal Classification of IBD ${ }^{(4)}$, and treated following published guidelines. Children with indeterminate colitis were excluded.

Forty-three cases of CD aged 7-18 years and twenty-six cases of UC aged 6-18 years were recruited. Median PCDAI score for CD was 10 (range 0-75), seventeen of which scored more than 15 (i.e. active disease) (Fig. 1). Median SCAI score for UC was 1 (range 0-13) (Fig. 2). Of the forty-three cases of $\mathrm{CD}$, fifteen were ileocaecal, twenty-two were ileocolonic and six had colitis only.

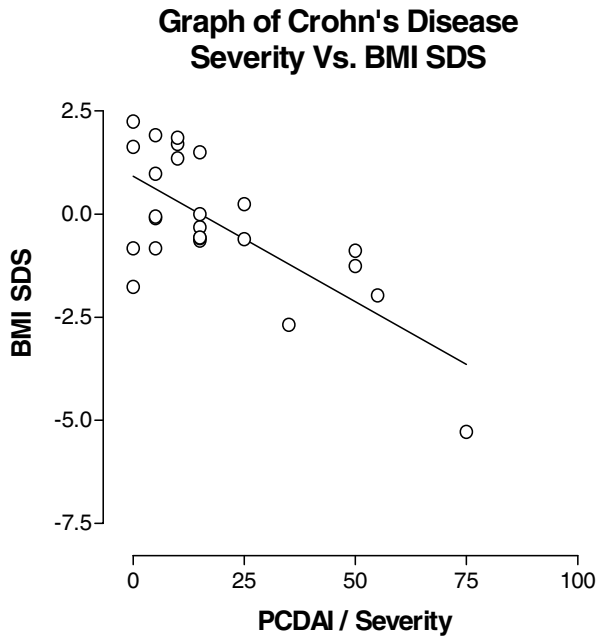

Fig. 1

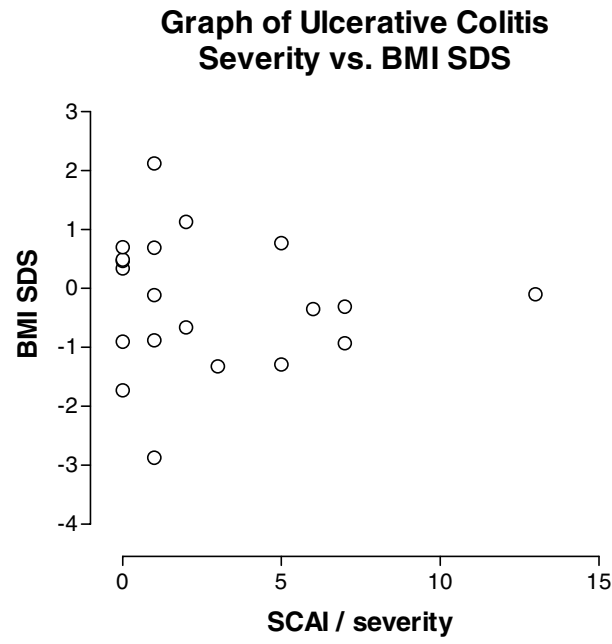

Fig. 2

There was a significant negative correlation (Spearman's correlation coefficient $r-0.45, P=0.003$ ) between disease activity and BMI standard deviation scores (SDS) in $\mathrm{CD}$, but not in $\mathrm{UC}(r 0.08, P=0.71)$. The strongest relationship was seen in ileocaecal CD. There was no relationship between disease activity and height SDS in either disease (CD, $r-0.11, P=0.49$; UC, $r-0.33, P=0.09$ ). Normal height SDS was preserved in all groups (CD, median - 0.675, range -3.16-6.89; UC, median -0.79 , range $-3.15-2.19$ ).

This study describes the relationship between disease activity and BMI in IBD. This adds to previous data demonstrating a nutritional deficit in $\mathrm{CD}$, but suggests this is less common in UC. BMI has a potential role as a clinical tool in childhood IBD and may highlight those in need of additional nutritional support. This data further supports the importance of nutritional assessment and management in the care of children with IBD.

1. Sawczenko A \& Sandhu B (2003) Arch Dis Child 88, 995-1000.

2. Hyams JS, Ferry GD, Mandel FS \& Gryboski JD (1991) J Paediatr Gastroenterol Nutr 12, 439-447.

3. Walmsley R, Ayres R, Pounder R \& Allan R (1998) Gut 43 (1), 29-32.

4. Silverberg MS, Satsangi J, Ahmad T, Arnott I, Berstein C, Brant MD et al. (2005) Can J gastroenterol 19 (suppl A). 5A-36A. 